\title{
Author Correction: Yolk sac macrophage progenitors traffic to the embryo during defined stages of development
}

\author{
C. Stremmel (1) 1,2, R. Schuchert ${ }^{1,2}$, F. Wagner ${ }^{1,2}$, R. Thaler ${ }^{1,2}$, T. Weinberger ${ }^{1,2}$, R. Pick2 , E. Mass (1) 3,4, \\ H.C. Ishikawa-Ankerhold ${ }^{1,2}$, A. Margraf ${ }^{2}$, S. Hutter ${ }^{5}$, R. Vagnozzi ${ }^{6}$, S. Klapproth ${ }^{7}$, J. Frampton ${ }^{8}$, S. Yona ${ }^{9}$, \\ C. Scheiermann², J.D. Molkentin ${ }^{6,10}$, U. Jeschke (iD ${ }^{5}$, M. Moser ${ }^{7}$, M. Sperandio ${ }^{2}$, S. Massberg $1,2,11$, \\ F. Geissmann (1) ${ }^{3} \&$ C. Schulz (10) $1,2,11$
}

Correction to: Nature Communications; https://doi.org/10.1038/s41467-017-02492-2; published online 8 Jan 2018.

This article contains errors in Figs. 5 and 6, for which we apologize. In Fig. 5f, the image 'E12.5 tail' was inadvertently replaced with a duplicate of the image 'E12.5 trunk' from the same panel. In Fig. 6d, the image 'E9.5/OH-TAM E8.5, embryo' was inadvertently replaced with a duplicate of the image 'E10.5/ OH-TAM E8.5, embryo' from Fig. 6b. The corrected versions of these figures appear below as Figs. 1 and 2. The raw data associated with these experiments can be accessed via the following link: https:/figshare.com/ articles/Yolk_sac_macrophage_progenitors_traffic_to_the_embryo_during_defined_stages_of_development/6930629.

Published online: 07 September 2018

\begin{abstract}
(c) Open Access This article is licensed under a Creative Commons Attribution 4.0 International License, which permits use, sharing, adaptation, distribution and reproduction in any medium or format, as long as you give appropriate credit to the original author(s) and the source, provide a link to the Creative Commons license, and indicate if changes were made. The images or other third party material in this article are included in the article's Creative Commons license, unless indicated otherwise in a credit line to the material. If material is not included in the article's Creative Commons license and your intended use is not permitted by statutory regulation or exceeds the permitted use, you will need to obtain permission directly from the copyright holder. To view a copy of this license, visit http://creativecommons.org/licenses/by/4.0/.
\end{abstract}

(C) The Author(s) 2018

\footnotetext{
${ }^{1}$ Medizinische Klinik und Poliklinik I, Klinikum der Universität, Ludwig-Maximilians-Universität, Marchioninistrasse 15, 81377 Munich, Germany. ${ }^{2}$ WalterBrendel-Center for Experimental Medicine, Ludwig-Maximilians-Universität, Marchioninistrasse 15, 81377 Munich, Germany. ${ }^{3}$ Immunology Program, Sloan Kettering Institute, Memorial Sloan Kettering Cancer Center, 1275 York Avenue, New York, NY 10065, USA. ${ }^{4}$ Developmental Biology of the Innate Immune System, LIMES-Institute, University of Bonn, Carl-Troll-Straße 31, 53115 Bonn, Germany. ${ }^{5}$ Klinik und Poliklinik für Frauenheilkunde und Geburtshilfe, Klinikum der Universität, Ludwig-Maximilians-Universität, Maistrasse 11, 80337 Munich, Germany. ${ }^{6}$ Department of Pediatrics, Cincinnati Children's Hospital Medical Center, 3333 Burnet Avenue, Cincinnati, OH 45229, USA. 7 Department of Molecular Medicine, Max Planck Institute of Biochemistry, Am Klopferspitz 18, 82152 Martinsried, Germany. ${ }^{8}$ Institute of Cancer and Genomic Sciences, College of Medical and Dental Sciences, University of Birmingham, Edgbaston, Birmingham B15 2TT, UK. ${ }^{9}$ Department of Immunology, The Weizmann Institute of Science, 234 Herzl Street, Rehovot 76100, Israel. ${ }^{10}$ Howard Hughes Medical Institute, Cincinnati Children's Hospital Medical Center, 3333 Burnet Avenue, Cincinnati, OH 45229, USA. ${ }^{11}$ DZHK (German Center for Cardiovascular Research), Partner Site Munich Heart Alliance, Biedersteiner Strasse 29, 80802 Munich, Germany. Correspondence and requests for materials should be addressed to C.S. (email: christian.schulz@med.uni-muenchen.de)
} 

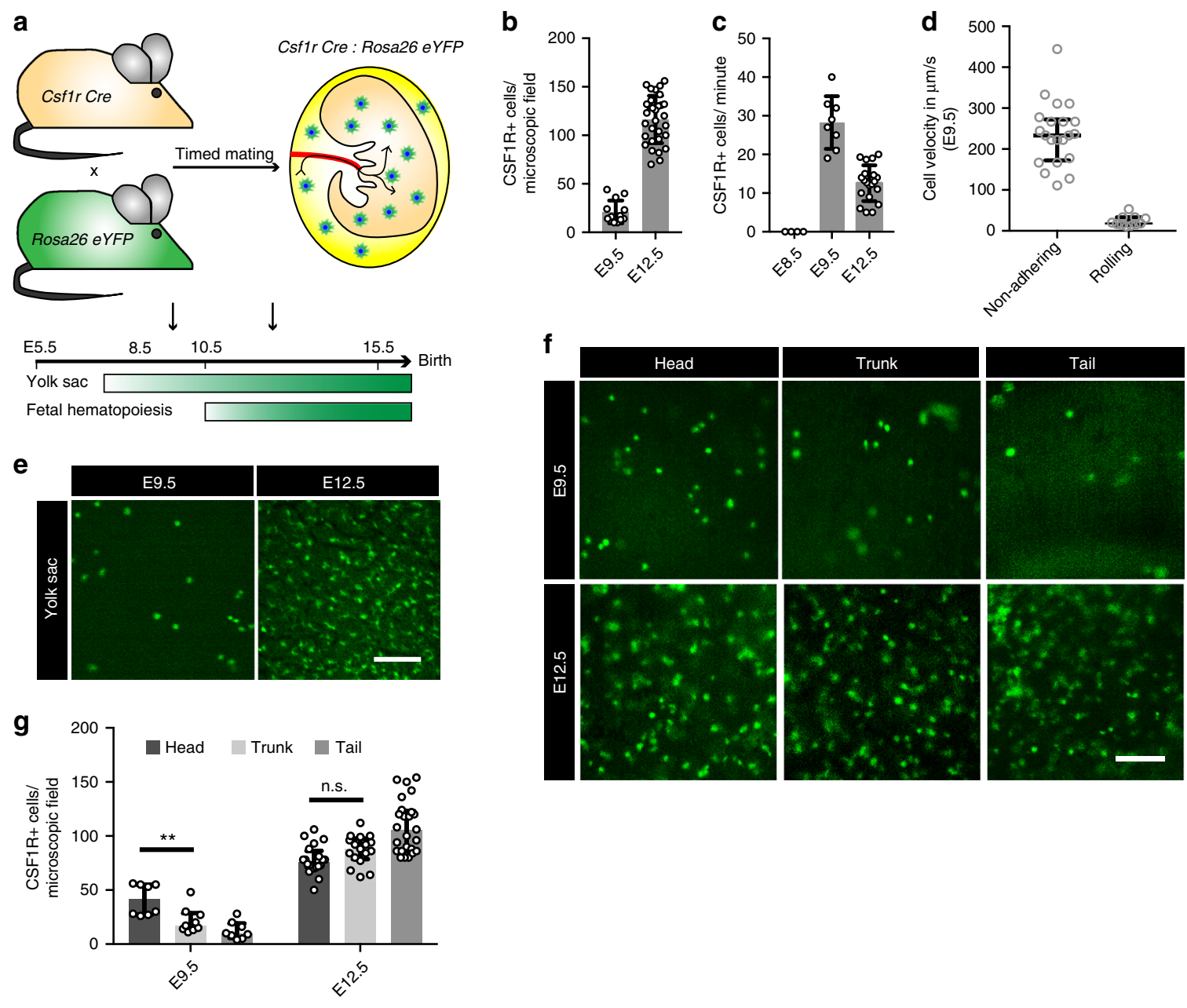

Fig. $1=$ 
a
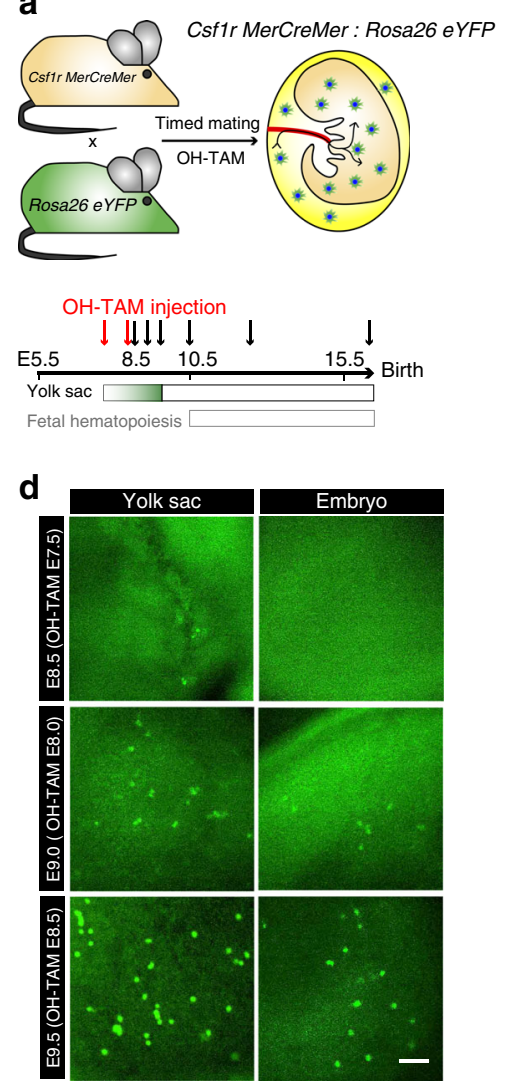

h

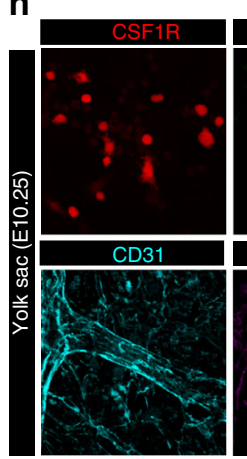

b
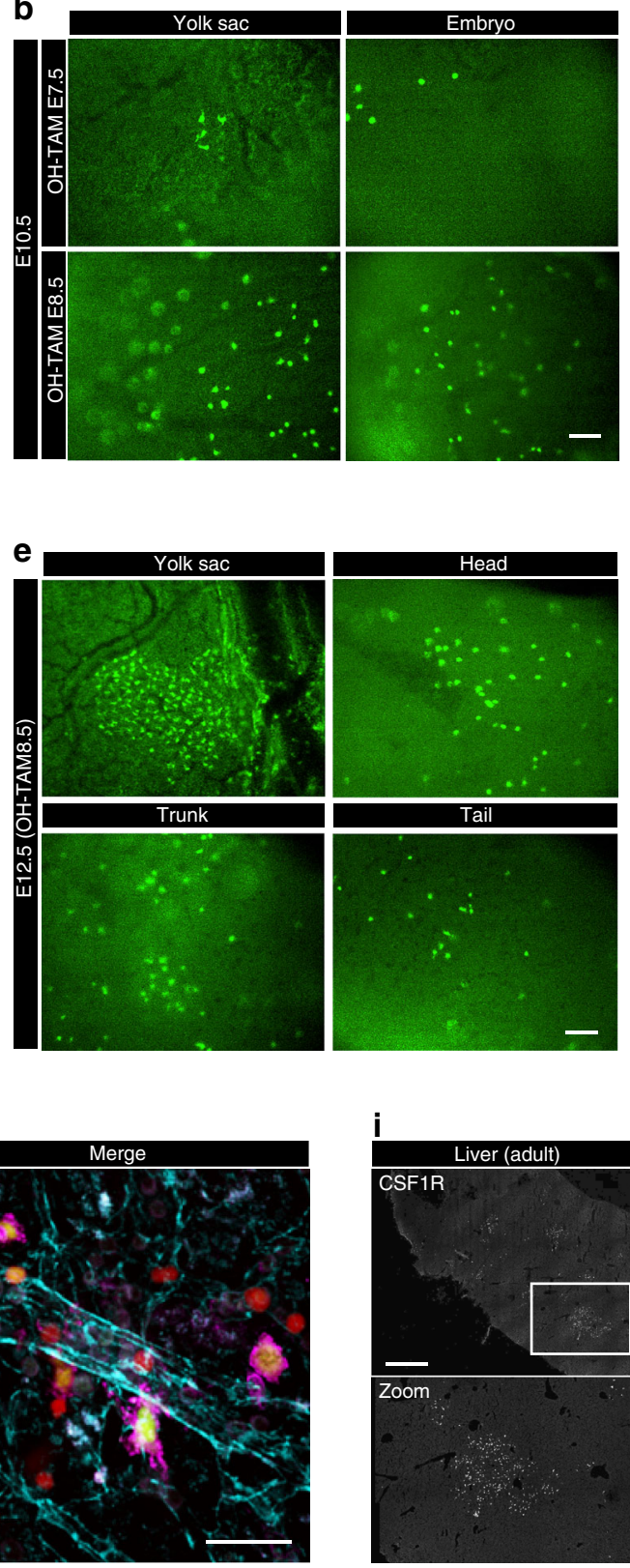

c

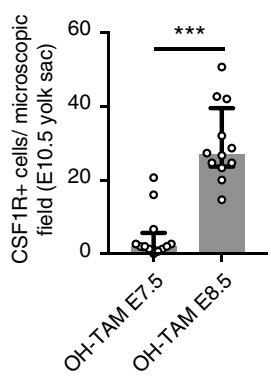

f

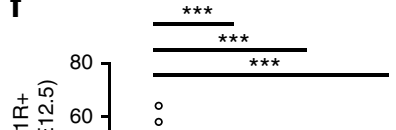

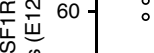

क⿺辶,

के

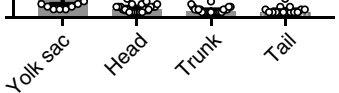
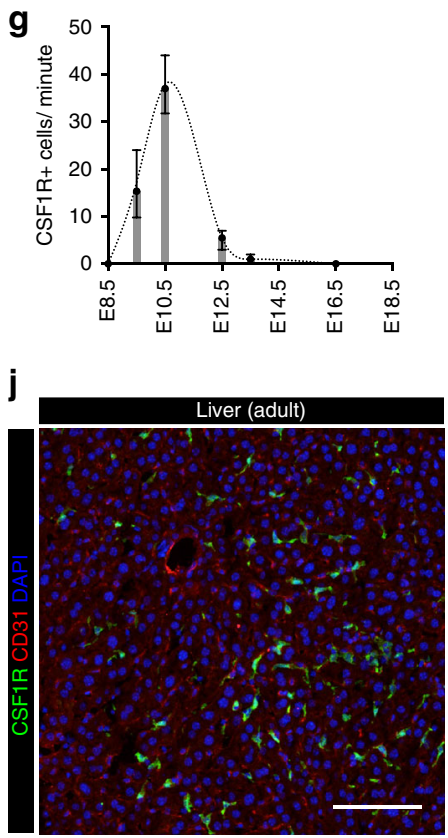

Fig. $2=$ 